Article

\title{
Investigation on Wear Behavior of Cryogenically Treated Ti-6Al-4V Titanium Alloy under Dry and Wet Conditions
}

\author{
Yonggang $\mathrm{Li}^{1,2, *}$, Xingfu Wang ${ }^{1}$, Shengqiang Yang ${ }^{1}$, Lifeng Hou ${ }^{3}$, Yinghui Wei ${ }^{3}$, \\ Zhongjie Zhang ${ }^{1}$ and Xiaoni Yang ${ }^{4}$ \\ 1 College of Mechanical and Vehicle Engineering, Taiyuan University of Technology, Taiyuan 030024, China \\ 2 Taiyuan Heavy Machinery Group Co., Ltd., Taiyuan 030024, China \\ 3 College of Materials Science and Engineering, Taiyuan University of Science and Technology, \\ Taiyuan 030024, China \\ 4 College of Water Resources Science and Engineering, Taiyuan University of Technology, \\ Taiyuan 030024, China \\ * Correspondence: liyonggang01@tyut.edu.cn; Tel.: +86-351-6018070
}

Received: 14 August 2019; Accepted: 31 August 2019; Published: 4 September 2019

\begin{abstract}
Titanium alloys are widely used in many fields because of their excellent comprehensive properties. However, its poor friction and wear properties limit its many potential applications. In general, the surface roughness of important parts manufactured by titanium alloy should meet certain requirements. As a low-cost and high-efficiency processing method, barrel finishing has been used for the surface finishing of titanium alloys. The main material removal mechanism of barrel finishing is micro-cutting/grinding, which is similar to the material wear mechanism under some conditions. In addition, titanium alloys are subjected to a low force in common surface finishing processes. Cryogenic treatment is a method that can improve the comprehensive properties of titanium alloys. Therefore, the friction and wear behavior of cryogenically treated Ti-6Al-4V titanium alloy (CT Ti alloy) and non-cryogenically treated Ti-6Al-4V titanium alloy (NT Ti alloy) at a low load and scratch speed was studied comparatively in this paper. The results show that the CT Ti alloy exhibits a lower friction coefficient and wear rate under both dry and wet wear conditions. Under wet conditions, the stabilized friction coefficient is lower than that under dry conditions. The stabilized friction coefficient of CT Ti alloy is 0.18 after reaching a stable wear stage under wet conditions. Under dry wear conditions, the NT Ti alloy mainly showed typical abrasive wear, heavy adhesion wear and oxidation wear characters. The wear mechanisms of CT Ti alloy are mainly abrasive wear, slight adhesion wear and oxidation wear. Under wet wear conditions, the wear mechanism of NT Ti alloy is abrasive wear and slight adhesion wear. After cryogenic treatment, the mechanism for CT Ti alloy is slight abrasive wear.
\end{abstract}

Keywords: Ti-6Al-4V alloy; cryogenic treatment; wear behavior

\section{Introduction}

Titanium alloys are favored in the production of aerospace parts due to their excellent comprehensive properties [1]. The demand and application of these alloys has been increased in other fields such as transportation, the petrochemical industry, biomedicine and daily life, etc. [2]. However, it also exposes many shortcomings and problems in the process of practical application. The shortcomings, like relatively low hardness, easy adhesion, poor thermal conductivity, low wear resistance, poor fatigue resistance and other defects, will greatly limit its popularization and application $[3,4]$. Some literatures show that the failure of titanium alloy parts is usually related to 
surface wear and corrosion [5]. Therefore, a comprehensive and in-depth study on the wear problem of titanium alloys is of great importance and significance. In view of the wear problem of titanium alloys, scholars have mainly focused on two aspects of research works. On the one hand, it is to improve the surface wear resistance of titanium alloy. On the other hand, it is to study the wear mechanism. In terms of wear resistance, researchers mainly apply the following means to improve the wear resistance of titanium alloys, such as particle reinforcement [6,7], laser cladding [8-10], surface plastic deformation [11-13], electrodeposition [14], ion implantation [15-17], anodic oxidation [18], magnetron sputtering [19], electrolytic/thermal oxidation [20,21], etc. In the aspect of wear mechanism, the main wear forms of titanium alloys were studied, including fretting wear, erosion wear, fatigue wear, adhesion wear, abrasive wear and corrosion wear. Sometimes the actual situation may be that some of these wear forms act simultaneously. Generally, with the change of actual working conditions, the influence degree of these wear forms also changes.

In recent years, the friction and wear properties of titanium alloys under dry, wet, corrosion, high temperature and lubrication conditions have attracted extensive attention of researchers. Straffelini et al. [22] investigated the dry friction and wear behavior of Ti-6Al-4V titanium alloy using the high speed steel as pairing material at room temperature. The results show that the wear mechanism transformation of the alloy occurs in the scratch speed range of $0.4-1.0 \mathrm{~m} / \mathrm{s}$, and the wear rate decreases with the increase of scratch speed. Liu et al. [23] studied the dry friction and wear properties of Ti-6Al-4V titanium alloy under low temperature and vacuum conditions. The results show that low temperature causes micro-cracks at wear marks at a low scratch speed. At a high scratch speed, micro-cracks do not occur. Cvijovic-Alagic et al. [24] studied the friction and wear properties of Ti-6Al-4V titanium alloy in simulated body fluids. It was found that the wear of Ti-6Al-4V alloy increased with the increase of scratch speed. Feng et al. [25] studied the effect of quenching treatment on the wear behavior of Ti-6Al-4V titanium alloy. The results show that there is no obvious change in the microstructure and hardness of Ti-6Al-4V titanium alloy after quenching at $750{ }^{\circ} \mathrm{C}$. When quenched above the temperature of the $\beta$ phase transition, the hardness of the alloy increases significantly from $400 \mathrm{HV}$ to $800 \mathrm{HV}$. However, the wear resistances of the quenched alloys with higher hardness decrease due to the change of wear mechanism from plastic deformation to brittle fracture. The effect of microstructure on wear behavior of Ti-6Al-4V titanium alloy was studied by Hadke et al. [26]. The results show that the hardness of the alloy increases and wear resistance decreases after quenching. The ability to resist abrasive wear is mainly affected by microstructure rather than hardness. The hardness of the original alloy is low, and the abrasive wear is mainly caused by micro-ploughing. The material removal mechanism of quenched samples is mainly caused by micro-cutting. Li et al. [27] studied the wear behavior of Ti-6Al-4V alloy in the sliding speed range of $0.5-4 \mathrm{~m} / \mathrm{s}$. The results showed that the wear rate changed in three stages: First slowly decreasing $(0.5-1.5 \mathrm{~m} / \mathrm{s})$, then rapidly increasing until the sliding speed reached $2.68 \mathrm{~m} / \mathrm{s}$, and then decreasing again $(2.68-4 \mathrm{~m} / \mathrm{s})$. When the sliding speed was $4 \mathrm{~m} / \mathrm{s}$, the wear rate was the lowest. The wear mechanisms in the above three stages are mainly delamination wear and oxidation wear at a low sliding speed, delamination wear at a medium speed and oxidation wear at a high speed. The adhesion wear, delamination wear and abrasive wear are the main wear types of Ti-6Al-4V alloy at $25-200{ }^{\circ} \mathrm{C}$ [28]. In the high temperature range of $400-500{ }^{\circ} \mathrm{C}$, oxidation wear is the main wear mechanism [28]. The experiments carried out by Cui et al. [29] show that the wear rate of Ti-6Al-4V alloy increases linearly with the load at $20^{\circ} \mathrm{C}$. When the temperature is $400^{\circ} \mathrm{C}$ and the load is $50-100 \mathrm{~N}$, the wear rate decreases gradually with the increase of the load. When the load is in the range of $100-200 \mathrm{~N}$, the wear rate increases with the increasing of the load. If the load is greater than $200 \mathrm{~N}$, the wear rate of the alloy increases rapidly. Frictional oxide plays a very good role in reducing wear during the wear process at $400{ }^{\circ} \mathrm{C}$. However, the investigation carried out by Wang et al. [30] showed that the dispersed frictional oxide had little antifriction effect when the load was in the range of 50-250 N and the temperature was in the range of $25-200{ }^{\circ} \mathrm{C}$. For Ti-6.5Al-3.5Mo- $1.5 \mathrm{Zr}-0.3 \mathrm{Si}$ alloy, frictional oxide takes a positive effect on reducing friction when the temperature is between $500{ }^{\circ} \mathrm{C}$ and $600{ }^{\circ} \mathrm{C}$. It is 
indicated that the chemical composition has a significant effect on the high temperature wear behavior of titanium alloys. Komotori et al. [31] found that the wear mechanism of Ti-6Al-4V alloy changed from abrasive wear to adhesive wear with the increase of friction speed. High friction speed hinders the formation of passive film at the wear mark and leads to pitting formation. Friction speed is an important factor affecting the wear failure process. Sahoo et al. [32] investigated the erosion wear resistance of Ti-6Al-4V titanium alloy. The impact velocity is the most important factor affecting the erosion wear of Ti-6Al-4V titanium alloy, and the change of microstructure has little effect on wear. This research team also studied the effect of friction factors on wear rate. When the load was between $50 \mathrm{~N}$ and $150 \mathrm{~N}$, the scratch speed was between $0.3 \mathrm{~m} / \mathrm{s}$ and $0.9 \mathrm{~m} / \mathrm{s}$ and the test time was between $30 \mathrm{~min}$ and $60 \mathrm{~min}$, and the effect of these friction factors on wear weight loss showed the following rules: Pressure (load) $>$ scratch speed $>$ test time $>$ microstructure. The oxidation wear occurs at a low scratch speed, while the delamination wear occurs at a high scratch speed [33]. In the aspect of friction reduction of titanium alloys, the friction and wear properties of titanium alloys can be improved by choosing suitable kinds of solid lubricants, particle sizes and concentrations [34,35].

Among many surface modification technologies, cryogenic treatment can improve the strength, toughness, wear resistance and corrosion resistance of titanium alloys comprehensively [36,37]. Moreover, the stability of mechanical processing can be improved by cryogenic treatment [38,39]. However, there are few studies on the friction and wear properties of cryogenically treated titanium alloys. Especially the investigations carried out under the conditions of low load and scratch speed. In fact, the wear conditions mentioned above are similar to those of barrel finishing. The mechanism of material removal of wear is also similar to that of barrel finishing and is micro-cutting/grinding. In various forms of the barrel finishing process, the force between the workpiece and abrasive particle is about $5 \mathrm{~N}$, and the relative velocity of the two parts is about $0.05 \mathrm{~m} / \mathrm{s}$ [40]. Therefore, the study of the friction and wear process and mechanism of titanium alloy under low load and scratch speed conditions will be helpful to improve the barrel finishing process of titanium alloy. It will provide a basis for realizing low cost and high efficiency surface processing for titanium alloy.

As a result, Ti-6Al-4V titanium alloy was used as the research material, and then it was placed in a liquid nitrogen cryogenic tank for cryogenic treatment. The friction and wear experiments of CT Ti alloy and NT Ti alloy were carried out under dry and wet wear conditions. The hardness, microstructure, wear rate, friction coefficient, surface morphology and wear mechanism of the samples before and after cryogenic treatment were systematically analyzed by means of a hardness tester, scanning electron microscopy (SEM), energy dispersive spectrometer (EDS) and 3D profiler.

\section{Materials and Methods}

\subsection{Material and Cryogenic Treatment}

Ti-6Al-4V titanium alloy was chosen as the research material in the experiment, and its composition is shown in Table 1. It was machined into a sample with a size of $20 \mathrm{~mm} \times 20 \mathrm{~mm} \times 10 \mathrm{~mm}$. Before the experiment, all Ti-6Al-4V titanium alloy samples were manually ground with sandpaper, then cleaned with ethanol and dried. The cryogenic treatment of titanium alloy was carried out in a CDW-196 liquid nitrogen cryogenic tank. Titanium alloy specimens were subjected to cryogenic treatment for $24 \mathrm{~h}$, and then recovered to room temperature for subsequent testing and analysis.

Table 1. Chemical composition of Ti-6Al-4V titanium alloy (wt. \%).

\begin{tabular}{rcccc}
\hline Ti & Al & V & Fe & C \\
\hline Bal. & 6.30 & 4.48 & 0.05 & 0.03 \\
\hline
\end{tabular}




\subsection{Wearing Test}

The reciprocating friction and wear experiments of Ti-6Al-4V titanium alloy under dry/wet conditions were carried out with a CFT-I multifunctional friction and wear tester (Lanzhou Zhongke Kaihua Technology Development Co., Ltd., Lanzhou, China) at room temperature. $\mathrm{ZrO}_{2}$ ceramic balls with a diameter of $3 \mathrm{~mm}$ were selected for the other friction pair material. The reciprocating length was $5 \mathrm{~mm}$. Five locations on the wear mark were selected for measurement, and the average value was taken as the wear amount of the wear mark. The wear rate was calculated as follows [41]:

$$
W r=\Delta V / L
$$

where $W r$ is the wear rate of the test material, $\mathrm{mm}^{3} / \mathrm{m} ; \Delta V$ is the wear volume, $\mathrm{mm}^{3}$; and $L$ is the wear distance, $\mathrm{m}$.

The main components of liquid media used in wet friction and wear experiments are sodium alcohol ether sulphate (AES), oleic acid, coconut oil fatty acid diethanolamin and primary alcohol ethoxylate (AEO). The liquid media formula shows as follows: Deionized water $1000 \mathrm{~mL}$, each liquid media $50 \mathrm{~mL}$. The formula is always used for barrel finishing of titanium alloy.

\subsection{Surface Analysis}

The microstructure of titanium alloy was analyzed by SEM (TESCAN, Brno, Czech Republic). Before the experiment, titanium alloy samples were ground with sandpaper, then polished and etched. The chemical etchant formula used in the experiment is a mixture of $90 \mathrm{~mL}$ deionized water, $2 \mathrm{~mL}$ hydrofluoric acid (HF) and $10 \mathrm{~mL}$ concentrated nitric acid $\left(\mathrm{HNO}_{3}\right)$. The surface hardness of the workpiece before and after cryogenic treatment was measured by an HMV-G21ST hardness tester (Shimadzu (Asia Pacific) Pte. Ltd., Kyoto, Japan). During the experiment, the load was $10 \mathrm{~N}$ and the testing time was $15 \mathrm{~s}$. Seven points were taken from each sample for measurement, and then the average value was taken as the final result. SEM was used to analyze the surface morphology of wear samples, and EDS was used to study the composition of wear marks and wear debris. The main working parameters of the SEM were an accelerating voltage of $30 \mathrm{kV}$ and a working distance of $15 \mathrm{~mm}$. The phase composition of the Ti-6Al-4V titanium alloy was analyzed via TD3600 X-ray diffraction (XRD, Dandong Tongda Science and Technology Co., Ltd., Dandong, China) equipped with a Cu target. The generator was set for $40 \mathrm{kV}$ and $30 \mathrm{~mA}$. Data was collected between $2 \theta=30^{\circ}$ and $80^{\circ}$ at $0.02^{\circ}$ intervals. The three-dimensional micro-morphology of wear marks on the surface of titanium alloy was observed by an SM-1000 laser copolymerization three-dimensional profilometer (Sixian Photoelectric technology (Shanghai) Co.,Ltd., Shanghai, China).

\section{Results and Discussion}

\subsection{Effect of Cryogenic Treatment on the Microstructure of Ti-6Al-4V Alloy}

Figure 1a shows the microstructures of the NT Ti alloy. It consists of two phases, the coarse $\alpha$ phase is dark gray, and fine $\beta$ phase is bright white. Figure $1 \mathrm{~b}$ shows the microstructures of the alloy after cryogenic treatment for $24 \mathrm{~h}$. Compared with the microstructures of the as-received material, the number and size of the $\beta$ phase of CT Ti alloy decrease slightly. This is consistent with the XRD results in Figure 2. Further analysis of XRD data shows that the content of the $\beta$ phase in the NT Ti alloy samples is $19.5 \%$, and that after cryogenic treatment for $24 \mathrm{~h}$, the content of the $\beta$ phase in the CT Ti alloy samples is $15 \%$. Cryogenic treatment results in the decrease of $\beta$ phase content in the alloys. This is consistent with the microstructure shown in Figure 1. The ambient temperature of the sample during cryogenic treatment is very low, which makes the volume of the material shrink. The investigation carried out by $\mathrm{Li}$ et al. shows that the pressure of Ti-6Al-4V titanium alloy specimens bear during cryogenic treatment $\left(-196{ }^{\circ} \mathrm{C}\right)$ is about $205 \mathrm{MPa} / \mathrm{cm}^{2}$ [42]. Under the action of enormous external force, part of the $\beta$ phase is transformed into a fine secondary $\alpha$ phase, which reduces the 
content of the $\beta$ phase to a certain extent [43]. At the same time, after cryogenic treatment, some defects and internal stress of the titanium alloy will be improved, which greatly enhances the stability of the material microstructure, thereby improving its mechanical properties [44].
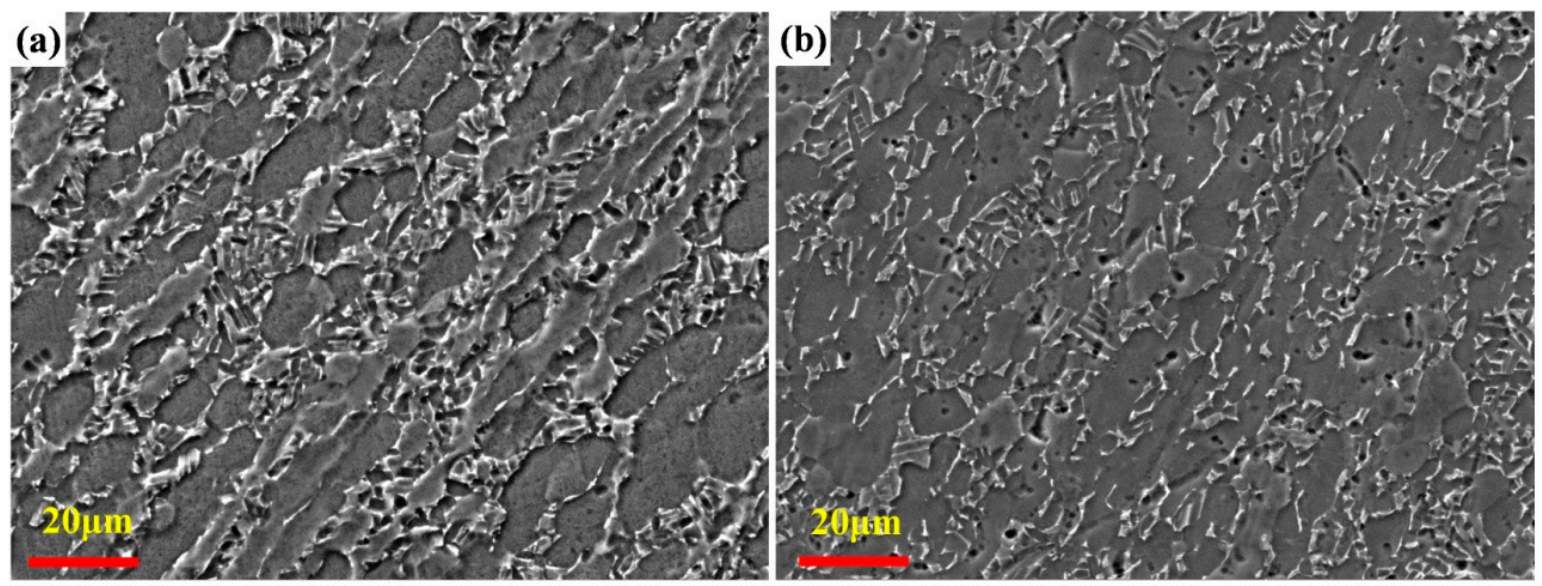

Figure 1. The microstructure of Ti-6Al-4V alloy: (a) as-received alloy (NT Ti alloy) and (b) after cryogenic treatment for $24 \mathrm{~h}$ (CT Ti alloy).

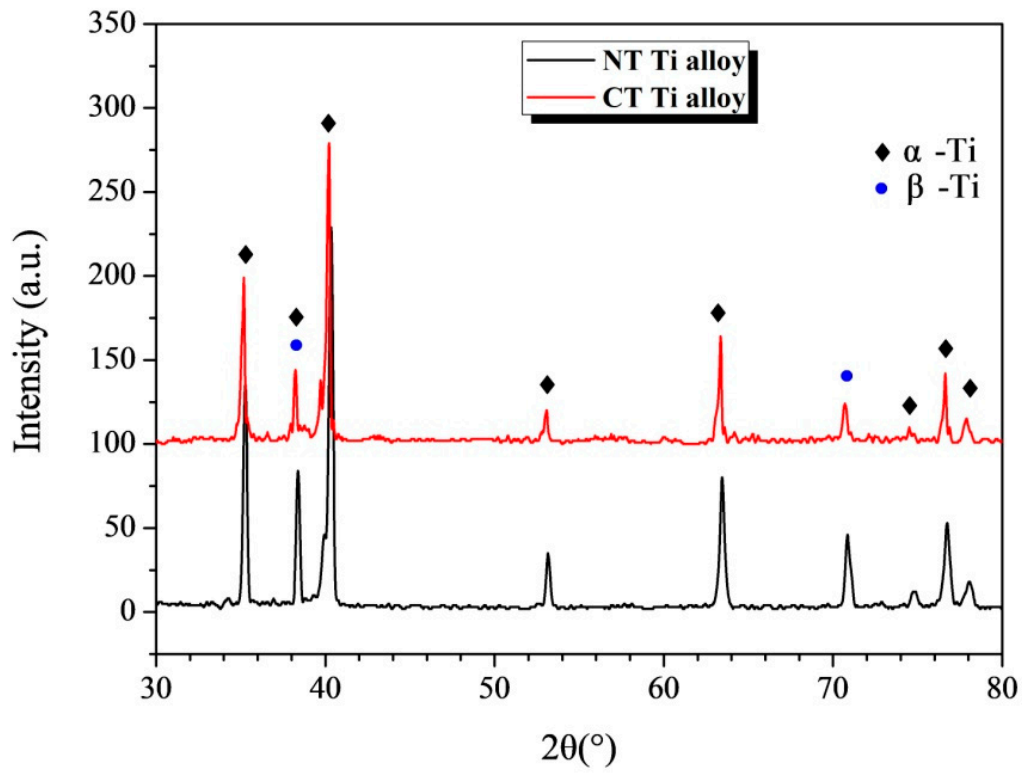

Figure 2. The X-ray diffraction (XRD) patterns of Ti-6Al-4V alloy with and without cryogenic treatment.

\subsection{Effect of Cryogenic Treatment on the Microhardness of Ti-6Al-4V Alloy}

Figure 3 is a histogram of the microhardness test result of Ti-6Al-4V titanium alloy at different cryogenic treatment times. The hardness of the NT Ti alloy sample is $341 \mathrm{HV}$. After 24 h cryogenic treatment, the microhardness of the titanium alloy sample increased to $368 \mathrm{HV}$, which was $7.9 \%$ higher than that of the untreated samples. In the two phases of Ti-6Al-4V alloy, the microhardness of the $\alpha$ phase is higher than that of the $\beta$ phase. Moreover, the compressive stress during cooling leads to the increase of dislocation density and a more stable lattice structure, which results in the increase of microhardness of the specimens after cryogenic treatment $[45,46]$. 


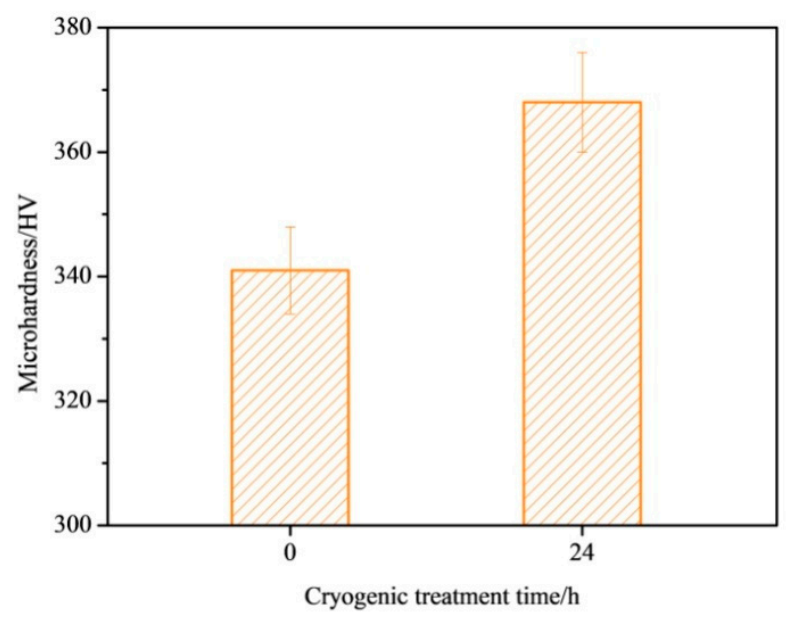

Figure 3. The microhardness of Ti-6Al-4V alloy with and without cryogenic treatment.

\subsection{Effect of Cryogenic Treatment on the Wear Behavior of Ti-6Al-4V Alloy}

\subsubsection{Effect of Cryogenic Treatment on the Friction Coefficient of Ti-6Al-4V Alloy}

Figure 4 shows the friction coefficient curves of NT Ti alloy and CT Ti alloy samples under dry and wet conditions with a load of $5 \mathrm{~N}$ and a sliding speed of $5 \mathrm{~cm} / \mathrm{s}$. From Figure $4 \mathrm{a}$, it was observed that the friction coefficient of the NT Ti alloy samples increased slowly with the prolongation of loading time within 0-6 min. When the loading time was extended to $6 \mathrm{~min}$, the coefficient value was about 0.55 . Then there was a sudden change, and the value increased to a certain extent. The friction coefficient gradually stabilized $7 \mathrm{~min}$ later. The friction coefficient fluctuated within $0-10 \mathrm{~min}$. The range of change is a little larger than that gained from the wet condition. The overall range of change was between 0.4-0.6. After $24 \mathrm{~h}$ of cryogenic treatment, it can be seen from Figure $4 \mathrm{~b}$ that the trend of friction coefficient change of CT Ti alloy specimens is similar to that of NT Ti alloy specimens in $3 \mathrm{~min}$. The friction coefficient of CT Ti alloy increases in 3-5 min. Then the coefficient decreases slightly and enters a stable stage in 5-10 min. It is indicated that the wear process also gradually enters a stable stage. The overall variation range of the friction coefficient was between 0.4 and 0.5 . The reasons for the change of the friction coefficient of titanium alloy samples are as follows: In the initial stage of friction, the adhesive wear is prone to occur on the surface of titanium alloy because the hardness of titanium alloy samples is less than that of $\mathrm{ZrO}_{2}$ balls. Moreover, the contact area of the two friction pair materials is small, resulting in large contact stress. The friction coefficient will increase to a certain extent after the adhesive wear occurs, and then tend to be stable [47].

Figure $4 \mathrm{c}, \mathrm{d}$ are the time-varying curves of the friction coefficients of Ti- $6 \mathrm{Al}-4 \mathrm{~V}$ titanium alloy before and after cryogenic treatment under wet conditions with a load of $5 \mathrm{~N}$ and a sliding speed of $5 \mathrm{~cm} / \mathrm{s}$. The results show that the friction coefficients of NT Ti alloy and CT Ti alloy samples are larger at the initial stage of wear. With the increase in loading time, the friction coefficients decreased obviously in both of the two groups of samples. Finally, the friction coefficients of NT Ti alloy samples tend to be 0.2 , while the friction coefficients of CT Ti alloy samples were stabilized at 0.18 . There is little difference in friction coefficient between the two groups of samples at the stable friction stage. However, the time needed to reach steady wear is different for these two kinds of specimens. After cryogenic treatment for $24 \mathrm{~h}$, the friction coefficient of the CT Ti alloy samples reached 0.18 after $3 \mathrm{~min}$. By comparison, the friction coefficient of the NT Ti alloy specimens was stabilized at 0.2 after $7 \mathrm{~min}$. At the same time, it can be seen that the friction coefficient of the NT Ti alloy samples suddenly decreases during the period of 4-5 $\mathrm{min}$, and then there is an obvious fluctuation. Some literatures show that even under lubrication conditions, adhesion wear of titanium alloys can occur [48], which is related to the contact state of friction pair materials in the initial stage of friction. With the progress of the experiment, a certain amount of debris is produced on the surface of the titanium alloy matrix, which plays the 
role of lubrication, isolation and support together with the liquid medium. As a result, the friction coefficient decreases to a certain extent. Therefore, it can be inferred that the adhesive wear degree of NT Ti alloy samples is more serious than that of CT Ti alloy samples under wet conditions. With the increase in loading time, the friction coefficients of NT Ti alloy samples and CT Ti alloy ones are all close to about 0.2 .

By comparing the variation in the friction coefficient after stabilization under dry and wet friction and wear conditions, it is found that the fluctuation range of the friction coefficient in dry conditions is larger than that in wet conditions. This is because the process from debris formation to debris leaving the friction system under dry conditions is relatively unstable compared with that under wet conditions. The variation range of the friction coefficient of CT Ti alloy is lower than that of NT Ti alloy, the coefficient value is smaller, and the friction and wear process is more stable. In this experiment, the friction and wear process of cryogenically treated samples is the most stable one under wet wear conditions.

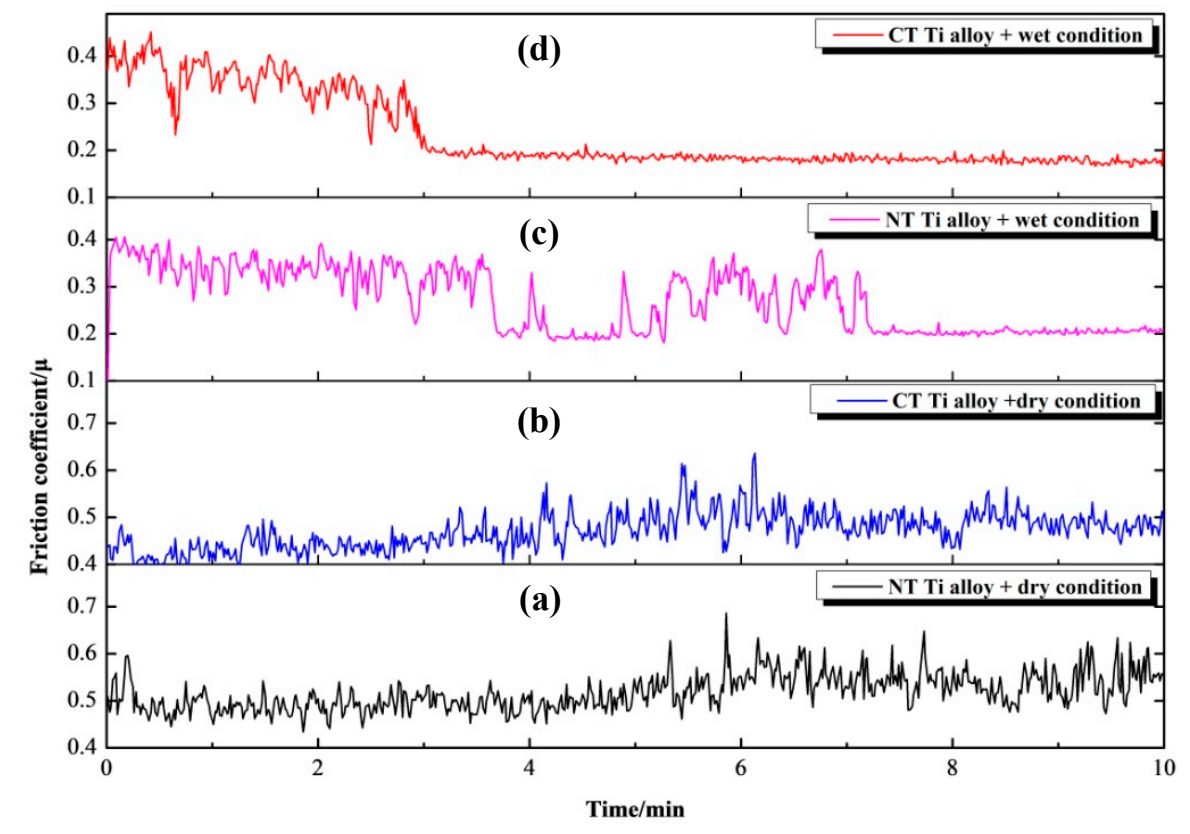

Figure 4. The friction coefficients of Ti-6Al-4V alloy with and without cryogenic treatment.

\subsubsection{Effect of Cryogenic Treatment on the Wear Rate of Ti-6Al-4V Alloy}

Figure 5 is a histogram of the wear rate of NT Ti alloy and CT Ti alloy under dry and wet wear conditions. The figure shows that, under dry wear conditions, the wear rate of CT Ti alloy samples is $31.5 \%$ lower than that of NT Ti alloy ones. After cryogenic treatment, the wear resistance of the titanium alloy under dry conditions can be improved. This is because, in general, higher hardness can give the material a better wear resistance $[49,50]$.

It can be seen from the figure that, under wet conditions, the wear rate of CT Ti alloy samples is $30.9 \%$ lower than that of NT Ti alloy samples. The wear resistance is obviously improved by cryogenic treatment. By comparison, it is found that the wear rates of CT Ti alloy samples under both dry and wet wear conditions are significantly lower than those of NT Ti alloy. The wear rate of the samples with and without cryogenic treatment is smaller under wet conditions, which is $54.3 \%$ and $53.6 \%$ lower than that under dry conditions, respectively. 


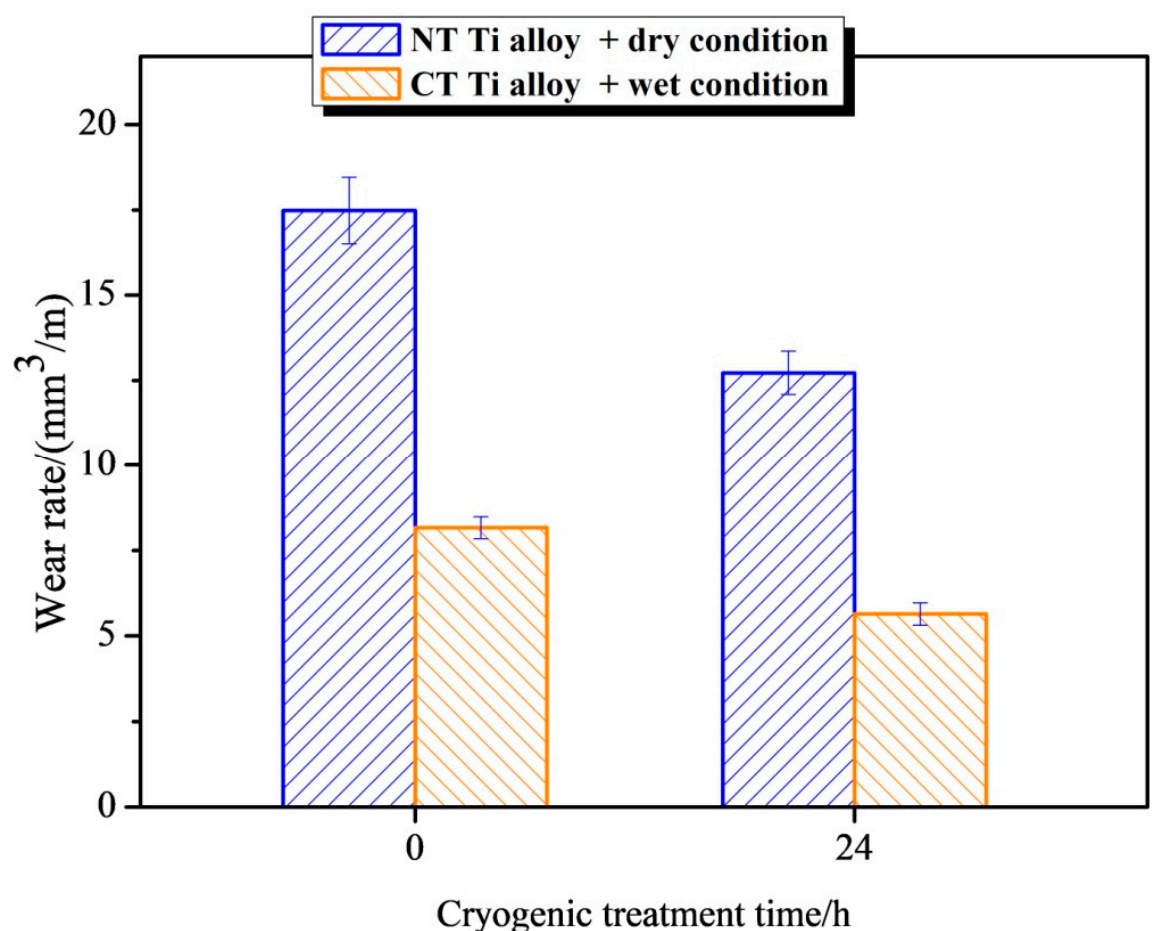

Figure 5. The wear rates of Ti-6Al-4V alloy with and without cryogenic treatment.

\subsubsection{Effect of Cryogenic Treatment on the Wear Morphologies and Mechanisms of Ti-6Al-4V Alloy}

Figure $6 \mathrm{a}-\mathrm{f}$ are the worn surface morphologies of Ti-6Al-4V alloy samples with and without cryogenic treatment under dry friction and wear conditions with a load of $5 \mathrm{~N}$ and a sliding speed of $5 \mathrm{~cm} / \mathrm{s}$. Figure $6 \mathrm{a}, \mathrm{b}$ show that the plough grooves on the surface of the NT Ti alloy samples are fine and dense, and a large number of white debris are attached to the surface of the wear marks. The layer-like protrusions in middle of the wear marks are formed due to the adhesion effect. Figure $6 \mathrm{~d}$,e reveal that the surface of the CT Ti alloy sample is relatively flat. The shallow plough grooves and slight adhesion marks distribute on the worn surface. Moreover, the width of the wear marks is narrower and shallower than that of NT Ti alloy. In addition, the wear characteristics of CT Ti alloy samples are similar to those in the literature [43].

Figure $6 \mathrm{c}, \mathrm{f}$ are 3D profiles of wear marks of NT Ti alloy and CT Ti alloy samples under dry friction. It can be seen that the wear marks of the titanium alloy matrix after the wear test are basically long pits. There are clearly visible plastic deformation zones turning to both sides of the pit edges. After cryogenic treatment for $24 \mathrm{~h}$, the depth of the wear scar profile of the titanium alloy is shallower than that of the non-cryogenic treated sample. The wear volume of the CT Ti alloy specimen is smaller, and the plastic deformation zones on both sides of the wear scar are not obvious. The results stated above indicate the wear rate of CT Ti alloy is lower than that of NT Ti alloy, which is basically consistent with the results shown in Figure 6.

Figure $6 \mathrm{~g}-\mathrm{l}$ are the worn surface morphologies of Ti-6Al-4V alloy samples under wet friction and wear conditions. There are obvious plough groove and slight adhesive wear on the surface of the NT Ti alloy sample shown in Figure 6g,h. The width of the wear mark is larger than that shown in Figure $6 j$. It can be seen from Figure 6j,k that the worn surface of the CT Ti alloy sample is relatively flat. Many shallow furrows distribute on the wear scar surface. There are no signs of adhesive wear. The width of the wear marks is narrower and the depth is shallower. 


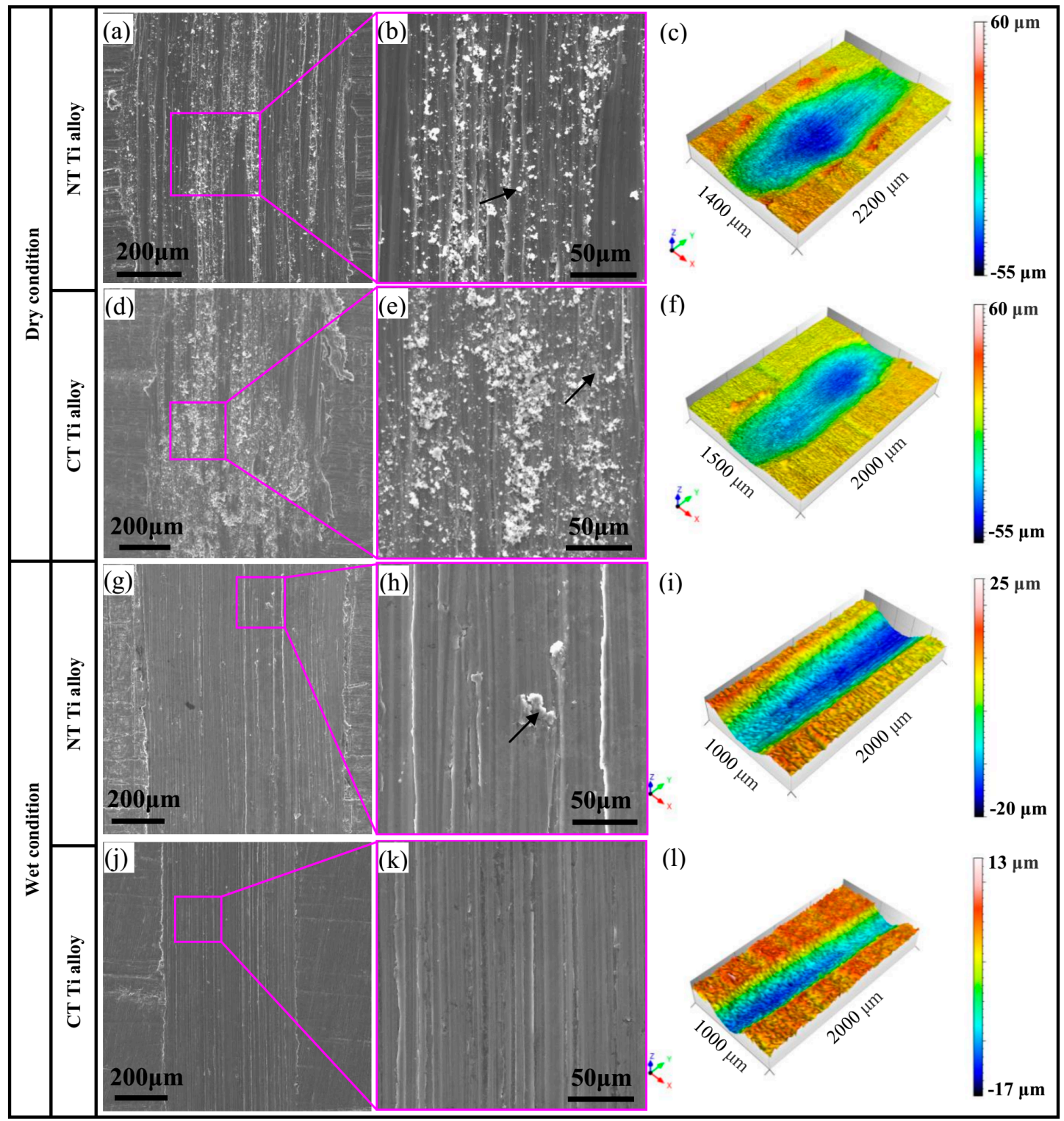

Figure 6. The wear morphologies of Ti-6Al-4V alloy before and after cryogenic treatment under dry/wet condition: $(\mathbf{a}-\mathbf{c})$ the SEM morphologies and the 3D morphology of NT Ti alloy under dry conditions; $(\mathbf{d}-\mathbf{f})$ the SEM morphologies and the 3D morphology of CT Ti alloy under dry conditions; ( $g-\mathbf{i})$ the SEM morphologies and the 3D morphology of NT Ti alloy under wet conditions; and ( $\mathbf{j}-\mathbf{1})$ the SEM morphologies and the 3D morphology of CT Ti alloy under wet conditions.

Figure $6 \mathrm{i}, 1$ are the 3D morphologies of wear marks of Ti-6Al-4V titanium alloy samples with and without cryogenic treatment under wet conditions. It can be seen from Figure $6 i$ that the wear scar morphology of the titanium alloy matrix after the wear test is basically the shape of the long pit under the condition of liquid lubrication. There are clearly visible plastic deformation zones turning to both sides of the pit edges. By comparison, the much slighter wear was observed on the surface of the CT Ti alloy. The main wear features are as follows: The depth and width of the wear marks are shallower, the wear volume is smaller and the plastic deformation zones on both sides of the wear marks are not obvious.

Compared with the surface morphology of wear marks under dry and wet wear conditions, it can be found that the surface of wear marks under wet wear conditions is smoother. There are fewer furrows, slighter adhesion phenomenon, smaller wear volume, shallower and narrower wear marks and a more stable wear process. It is indicated that the wear resistance of the alloy is higher 
under wet condition. The CT Ti alloy samples have better wear resistance than NT Ti alloy ones under dry/wet conditions. This is because cryogenic treatment can improve the hardness and processing stability of Ti-6Al-4V titanium alloy, and these factors will help to improve its wear resistance.

The results of the chemical composition analysis for typical wear areas are shown in Figure 7. Figure $7 \mathrm{a}$ is the EDS result of white debris in Figure $6 \mathrm{~b}$. The results show that there are $\mathrm{Zr}$ and $\mathrm{O}$ elements in the debris. The transfer of the $\mathrm{Zr}$ element to debris indicates the occurrence of adhesive wear. Figure $7 \mathrm{~b}$ is an analysis of the pale area in Figure 6e that will be peeled off from the matrix. This area contains not only the element of the matrix, but also the O element. EDS analysis was carried out on the middle position of the dark gray area to be peeled off from the matrix in Figure $6 \mathrm{~h}$. The results showed that the main composition was the elements in the matrix (Figure 7c). According to the comprehensive analysis of Figures 6 and 7, the wear mechanism of NT Ti alloy samples are the combination of typical abrasive wear, heavy adhesion wear and slight oxidation wear under dry wear condition. The wear mechanism for CT Ti alloy samples are abrasive wear, slight adhesion wear and oxidation wear. The results show that the wear of CT Ti alloy is slighter than that of NT Ti alloy, and the wear process is more stable. The wear resistance of Ti-6Al-4V titanium alloy is improved by cryogenic treatment. Under wet wear conditions, the wear mechanism of samples without cryogenic treatment is abrasive wear and slight adhesion wear. After cryogenic treatment, the abrasive wear is the main mechanism for CT Ti alloy.

When the hardness of the material itself is high, the abrasive wear is prone to occur, and when the hardness is low, adhesive wear will be appearing [51]. The higher the contact stress, the easier the adhesive wear will occur [52]. If the contact stress is high and exceeds a $1 / 3$ of the hardness of the material, adhesive wear will easily occur. In addition, cryogenic treatment results in more dislocations in titanium alloy samples [42,45], which improves the resistance to micro-plastic deformation. These factors together lead to slighter adhesion wear of CT Ti alloy samples. If the scratching speed is low, the oxidation wear of the titanium alloy will also occur at a low temperature [33]. Therefore, under dry friction and wear conditions, abrasive wear, adhesion wear and slight oxidation wear will occur on titanium alloys. In the initial stage of wear, due to the small contact area and large contact stress between the friction pair, under the action of shear stress in the friction process, the materials on the surface of the titanium alloy and the surface of the $\mathrm{ZrO}_{2}$ ball transfer mutually. The material transfer process is mainly from the surface of the titanium alloy sample to that of the $\mathrm{ZrO}_{2}$ ball. At this time, micro-convex bodies will be formed on the $\mathrm{ZrO}_{2}$ ball, and these micro-convex bodies will have a serious ploughing effect on the titanium alloy samples. Therefore, the friction coefficient increases slowly in the initial stage of friction. As the friction process proceeds, the micro-convex body will harden and fall off from the $\mathrm{ZrO}_{2}$ ball, so the composition element of the $\mathrm{ZrO}_{2}$ ball will be detected in the debris. In the later stage of friction, the material transfer process between two friction pair materials and the formation of debris gradually stabilize, so the friction coefficient gradually stabilizes at a higher value. Cryogenic treatment makes the Ti-6Al-4V titanium alloy more uniform in microstructure and higher in hardness, which leads to CT Ti alloy having a better wear resistance.

Under the wet condition, the liquid medium can isolate oxygen to a great extent, so the wear mechanism of titanium alloy is mainly abrasive wear and adhesive wear. Under the condition of wet wear, because of the existence of liquid medium, the temperature between friction pairs is low, and the material transfer between friction pairs is difficult, which restrains the occurrence of adhesive wear. Therefore, under the wet condition, the friction coefficient of titanium alloy samples will gradually decrease, and then the wear process will reach a stable state. The plough grooves on the NT Ti alloy surface are wider and mainly formed by the ploughing effect from the micro-convex body. These micro-convex bodies are mainly formed by the titanium alloy material transferred to the $\mathrm{ZrO}_{2}$ ball. The plough grooves on the surface of CT Ti alloy are fine, which is mainly induced by the micro-cutting process. The micro-convex body on the surface of the $\mathrm{ZrO}_{2}$ ball plays an important role in the process. In conclusion, cryogenic treatment can reduce the adhesive wear of Ti-6Al-4V titanium alloy under dry/wet friction conditions. 
In this paper, the effect of cryogenic treatment on the friction and wear properties of Ti-6Al-4V titanium alloy is studied. The friction process can be regarded as a single abrasive particle scratching on the surface of titanium alloys. The force and relative velocity between the workpiece and abrasive particles are the two most important parameters in the process of barrel finishing. The main parameters of this experiment are obtained in the actual finishing process of Ti-6Al-4V titanium alloy. The experimental results show that the friction process of CT Ti alloy can rapidly stabilize under wet friction and wear conditions. It is indicated that the friction process of titanium alloy under the above conditions is more predictable and controllable. It means that through a reasonable experimental design and experimental simplification, the parameters needed for actual processing can be explored with lower costs and higher efficiency.
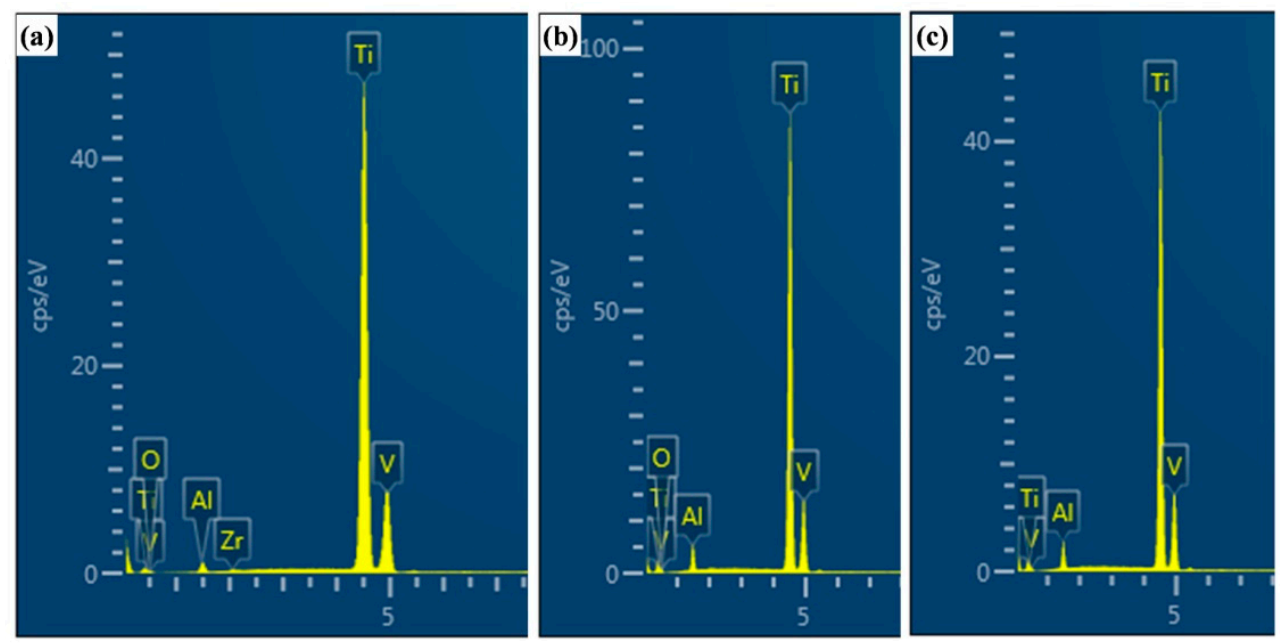

Figure 7. EDS results of a typical area on the worn surface in Figure 6.

\section{Conclusions}

The friction and wear behavior of Ti-6Al-4V titanium alloy with and without cryogenic treatment under a low load $(5 \mathrm{~N})$, low scratch speed $(5 \mathrm{~cm} / \mathrm{s})$ and dry/wet conditions were studied. The main conclusions are as follows:

(1) Cryogenic treatment leads to the transformation of part of the $\beta$ phase into a secondary $\alpha$ phase in the microstructure of Ti-6Al-4V titanium alloy. The comprehensive effects of the cryogenic treatment result in improved hardness, which is helpful to enhance the friction and wear properties of the titanium alloy.

(2) After cryogenic treatment, the Ti-6Al-4V titanium alloy exhibits a lower friction coefficient and wear rate under both dry and wet wear conditions. Under wet conditions, the friction coefficient after stabilization is lower than that under dry conditions. After cryogenic treatment, the friction coefficient of Ti-6Al-4V alloy is 0.18 after reaching a stable wear stage under wet conditions. The time required for the CT Ti alloy to reach the stable wear stage is obviously shorter than that for the NT Ti alloy. Cryogenic treatment makes CT Ti alloy have a more uniform microstructure and higher microhardness than NT Ti alloy, so that the former can enter a stable friction stage in a shorter time.

(3) Under dry wear conditions, the wear mechanism of NT Ti alloy samples is typical abrasive wear, heavy adhesion wear and slight oxidation wear. The wear mechanism of CT Ti alloy samples is abrasive wear, slight oxidation wear and adhesion wear. Under wet wear conditions, the wear mechanism of NT Ti alloy samples is abrasive wear and slight adhesion wear. After cryogenic treatment, the mechanism is changed to slight abrasive wear. Cryogenic treatment improves the microhardness of Ti-6Al-4V titanium alloy and reduces its tendency of adhesion wear. With the participation of a liquid medium, the wear process of Ti-6Al-4V titanium alloy can stabilize quickly. The combination 
use of a cryogenic treatment and a liquid medium will make the wear process of titanium alloy more predictable and controllable.

Author Contributions: Funding acquisition, Y.L.; investigation, X.W., L.H., Y.W. and Z.Z.; methodology, S.Y.; writing—original draft, Y.L. and X.Y.

Funding: This research was funded by the National Natural Science Foundation of China (No. 51705352) and the China Postdoctoral Science Foundation (No. 2018M641682) supported this work.

Conflicts of Interest: The authors declare no conflict of interest.

\section{References}

1. de Castro, M.C.B.; Couto, A.A.; Almeida, G.F.C.; Massi, M.; de Lima, N.B.; Sobrinho, A.D.; Castagnet, M.; Xavier, G.L.; Oliveira, R.R. The effect of plasma nitriding on the fatigue behavior of the Ti-6Al-4V alloy. Materials 2019, 12, 520. [CrossRef] [PubMed]

2. Hollander, D.A.; von Walter, M.; Wirtz, T.; Sellei, R.; Schmidt-Rohlfing, B.; Paar, O.; Erli, H.J. Structural, mechanical and in vitro characterization of individually structured Ti-6Al-4V produced by direct laser forming. Biomaterials 2006, 27, 955-963. [CrossRef] [PubMed]

3. Chen, C.; Feng, X.F.; Shen, Y.F. A comparative investigation on bi-layer Al-Cr/Al-Si coating and mono-layer Al-Cr-Si coating synthesized on Ti-6Al-4V alloy substrate by mechanical alloying method. J. Alloy. Compd. 2017, 708, 639-651. [CrossRef]

4. Kikuchi, S.; Yoshida, S.; Ueno, A. Improvement of fatigue properties of Ti-6Al-4V alloy under four-point bending by low temperature nitriding. Int. J. Fatigue 2019, 120, 134-140. [CrossRef]

5. Rahmati, B.; Sarhan, A.A.D.; Basirun, W.J.; Abas, W.A.B.W. Ceramic tantalum oxide thin film coating to enhance the corrosion and wear characteristics of Ti-6Al-4V alloy. J. Alloy. Compd. 2016, 676, 369-376. [CrossRef]

6. Hu, R.H.; Dewidar, M.M.; Kim, H.J.; Lim, J.K. Effect of $\mathrm{Si}_{3} \mathrm{~N}_{4}$ addition on the mechanical properties, microstructures, and wear resistance of Ti-6Al-4V alloy. J. Mater. Sci. Technol. 2007, 23, 257-261.

7. Patil, A.S.; Hiwarkar, V.D.; Verma, P.K.; Khatirkar, R.K. Effect of $\mathrm{TiB}_{2}$ addition on the microstructure and wear resistance of Ti-6Al-4V alloy fabricated through direct metal laser sintering (DMLS). J. Alloy. Compd. 2019, 777, 165-173. [CrossRef]

8. Yang, Y.L.; Zhang, D.; Yan, W.; Zheng, Y.R. Microstructure and wear properties of TiCN/Ti coatings on titanium alloy by laser cladding. Opt. Laser Eng. 2010, 48, 119-124. [CrossRef]

9. Weng, F.; Yu, H.J.; Chen, C.Z.; Liu, J.L.; Zhao, L.J.; Dai, J.J.; Zhao, Z.H. Effect of process parameters on the microstructure evolution and wear property of the laser cladding coatings on Ti-6Al-4V alloy. J. Alloy. Compd. 2017, 692, 989-996. [CrossRef]

10. Wang, H.J.; Chen, T.; Cong, W.L.; Liu, D.F. Laser cladding of Ti-based ceramic coatings on Ti6Al4V alloy: Effects of $\mathrm{CeO}_{2}$ nanoparticles additive on wear performance. Coatings 2019, 9, 109. [CrossRef]

11. Amanov, A.; Cho, I.S.; Kim, D.E.; Pyun, Y.S. Fretting wear and friction reduction of CP titanium and Ti-6Al-4V alloy by ultrasonic nanocrystalline surface modification. Surf. Coat. Technol. 2012, 207, 135-142. [CrossRef]

12. Revankar, G.D.; Shetty, R.; Rao, S.S.; Gaitonde, V.N. Wear resistance enhancement of titanium alloy (Ti-6A1-4V) by ball burnishing process. J. Mater. Res. Technol. 2017, 6, 13-32. [CrossRef]

13. Li, G.; Qu, S.G.; Pan, Y.X.; Li, X.Q. Effects of the different frequencies and loads of ultrasonic surface rolling on surface mechanical properties and fretting wear resistance of HIP Ti-6Al-4V alloy. Appl. Surf. Sci. 2016, 389, 324-334. [CrossRef]

14. Lee, C.K. Corrosive wear and mechanical properties of $\mathrm{Ni} / \mathrm{Al}_{2} \mathrm{O}_{3}$ micro- and nanoparticulates-reinforced coatings on Ti-6Al-4V alloy. Tribol. Trans. 2012, 55, 640-651. [CrossRef]

15. Mohan, L.; Anandan, C. Wear and corrosion behavior of oxygen implanted biomedical titanium alloy Ti-13Nb-13Zr. Appl. Surf. Sci. 2013, 282, 281-290. [CrossRef]

16. Taktak, S.; Akbulut, H. Dry wear and friction behaviour of plasma nitride Ti-6AL-4V alloy after explosive shock treatment. Tribol. Int. 2007, 40, 423-432. [CrossRef]

17. Tsuji, N.; Tanaka, S.; Takasugi, T. Effects of combined plasma-carburizing and shot-peening on fatigue and wear properties of Ti-6Al-4Valloy. Surf. Coat. Technol. 2009, 203, 1400-1405. [CrossRef] 
18. Habazaki, H.; Onodera, T.; Fushimi, K.; Konno, H.; Toyotake, K. Spark anodizing of beta-Ti alloy for wear-resistant coating. Surf. Coat. Technol. 2007, 201, 8730-8737. [CrossRef]

19. Amanov, A.; Watabe, T.; Tsuboi, R.; Sasaki, S. Fretting wear and fracture behaviors of Cr-doped and non-doped DLC films deposited on Ti-6Al-4V alloy by unbalanced magnetron sputtering. Tribol. Int. 2013, 62, 49-57. [CrossRef]

20. Cheng, Y.L.; Wu, X.Q.; Xue, Z.G.; Matykina, E.; Skeldon, P.; Thompson, G.E. Microstructure, corrosion and wear performance of plasma electrolytic oxidation coatings formed on Ti-6Al-4V alloy in silicate-hexametaphosphate electrolyte. Surf. Coat. Technol. 2013, 217, 129-139. [CrossRef]

21. Zhou, Y.; Zhang, Q.Y.; Liu, J.Q.; Cui, X.H.; Mo, J.G.; Wang, S.Q. Wear characteristics of a thermally oxidized and vacuum diffusion heat treated coating on Ti-6Al-4V alloy. Wear 2015, 344, 9-21. [CrossRef]

22. Straffelini, G.; Molinari, A. Dry sliding wear of Ti-6Al-4V alloy as influenced by the counterface and sliding conditions. Wear 1999, 236, 328-338. [CrossRef]

23. Liu, Y.; Yang, D.Z.; He, S.Y.; Ye, Z.Y. Dry sliding wear of Ti-6Al-4V alloy at low temperature in vacuum. Space Technol. Proc. 2006, 6, 309-316.

24. Cvijovic-Alagic, I.; Cvijovic, Z.; Mitrovic, S.; Panic, V.; Rakin, M. Wear and corrosion behaviour of Ti-13Nb-13Zr and Ti-6Al-4V alloys in simulated physiological solution. Corros. Sci. 2011, 53, 796-808. [CrossRef]

25. Feng, C.; Khan, T.I. The effect of quenching medium on the wear behaviour of a Ti-6Al-4V alloy. J. Mater. Sci. 2008, 43, 788-792. [CrossRef]

26. Hadke, S.; Khatirkar, R.K.; Shekhawat, S.K.; Jain, S.; Sapate, S.G. Microstructure evolution and abrasive wear behavior of Ti-6Al-4V alloy. J. Mater. Eng. Perform. 2015, 24, 3969-3981. [CrossRef]

27. Li, X.X.; Zhou, Y.; Ji, X.L.; Li, Y.X.; Wang, S.Q. Effects of sliding velocity on tribo-oxides and wear behavior of Ti-6Al-4V alloy. Tribol. Int. 2015, 91, 228-234. [CrossRef]

28. Mao, Y.S.; Wang, L.; Chen, K.M.; Wang, S.Q.; Cui, X.H. Tribo-layer and its role in dry sliding wear of Ti-6Al-4V alloy. Wear 2013, 297, 1032-1039. [CrossRef]

29. Cui, X.H.; Mao, Y.S.; Wei, M.X.; Wang, S.Q. Wear characteristics of Ti-6Al-4V alloy at $20-400{ }^{\circ} \mathrm{C}$. Tribol. Trans. 2012, 55, 185-190. [CrossRef]

30. Wang, L.; Zhang, Q.Y.; Li, X.X.; Cui, X.H.; Wang, S.Q. Dry Sliding Wear Behavior of Ti-6.5Al-3.5Mo-1.5Zr-0.3Si Alloy. Metall. Mater. Trans. A 2014, 45A, 2284-2296. [CrossRef]

31. Komotori, J.; Hisamori, N.; Ohmori, Y. The corrosion/wear mechanisms of Ti-6Al-4V alloy for different scratching rates. Wear 2007, 263, 412-418. [CrossRef]

32. Sahoo, R.; Mantry, S.; Sahoo, T.K.; Mishra, S.; Jha, B.B. Effect of microstructural variation on erosion wear behavior of Ti-6Al-4V Alloy. Tribol. Trans. 2013, 56, 555-560. [CrossRef]

33. Sahoo, R.; Jha, B.B.; Sahoo, T.K.; Sahoo, D. Effect of Microstructural variation on dry sliding wear behavior of Ti-6Al-4V alloy. J. Mater. Eng. Perform. 2014, 23, 2092-2102. [CrossRef]

34. Gunda, R.K.; Narala, S.K.R. Tribological studies to analyze the effect of solid lubricant particle size on friction and wear behaviour of Ti-6Al-4V alloy. Surf. Coat. Technol. 2016, 308, 203-212. [CrossRef]

35. Zhang, Q.Y.; Wang, S.Q.; Zhou, Y.; Chen, K.M.; Wang, L.; Cui, X.H. Artificial oxide-containing tribo-layers and their effect on wear performance of Ti-6Al-4V alloy. Tribol. Int. 2017, 105, 334-344. [CrossRef]

36. Gu, K.X.; Zhang, H.; Zhao, B.; Wang, J.J.; Zhou, Y.; Li, Z.Q. Effect of cryogenic treatment and aging treatment on the tensile properties and microstructure of Ti-6Al-4V alloy. Mater. Sci. Eng. A 2013, 584, 170-176. [CrossRef]

37. Zhou, J.Z.; Li, J.; Huang, S.; Sheng, J.; Meng, X.K.; Sun, Q.; Sun, Y.H.; Xu, G.F.; Sun, Y.J.; Li, H.T. Influence of cryogenic treatment prior to laser peening on mechanical properties and microstructural characteristics of TC6 titanium alloy. Mater. Sci. Eng. A 2018, 718, 207-215. [CrossRef]

38. Ahmed, L.S.; Kumar, M.P. Cryogenic drilling of Ti-6Al-4V alloy under liquid nitrogen cooling. Mater. Manuf. Process. 2016, 31, 951-959. [CrossRef]

39. Gill, S.S.; Singh, J. Effect of deep cryogenic treatment on machinability of titanium alloy (Ti-6246) in electric discharge drilling. Mater. Manuf. Process. 2010, 25, 378-385. [CrossRef]

40. Chen, Y.N.; Li, W.H.; Li, X.H.; Wang, X.Z. Force test and analysis of abrasive in spindle barrel finishing process. China Surf. Eng. 2017, 30, 33-40.

41. Huang, T.D.; Jiang, Li.; Zhang, C.L.; Jiang, H.; Lu, Y.P.; Li, T.J. Effect of carbon addition on the microstructure and mechanical properties of CoCrFeNi high entropyalloy. Sci. China Technol. Sc. 2018, 61, 117-123. [CrossRef] 
42. Li, G.R.; Qin, T.; Fei, A.G.; Wang, H.M.; Zhao, Y.T.; Chen, G.; Kai, X.Z. Performance and microstructure of TC4 titanium alloy subjected to deep cryogenic treatment and magnetic field. J. Alloy. Compd. 2019, 802, 50-69. [CrossRef]

43. Gu, K.X.; Wang, J.J.; Zhou, Y. Effect of cryogenic treatment on wear resistance of Ti-6Al-4V alloy for biomedical applications. J. Mech. Behav. Biomed. 2014, 30, 131-139. [CrossRef] [PubMed]

44. Singla, A.K.; Singh, J.; Kumar, P.; Kumar, A.; Sharma, V. Effect of cryogenic treatment on the wear resistance and microstructure of Ti-6Al-4V. Indian J. Eng. Mater. Sci. 2018, 25, 243-249.

45. Xu, L.Y.; Zhu, J.; Jing, H.Y.; Zhao, L.; Lv, X.Q.; Han, Y.D. Effects of deep cryogenic treatment on the residual stress and mechanical properties of electron-beam-welded Ti-6Al-4V joints. Mater. Sci. Eng. A 2016, 676, 503-510. [CrossRef]

46. Graca, S.; Colaco, R.; Carvaloh, R.A.; Vilar, R. Determination of dislocation density from hardness measurements in metals. Mater. Lett. 2008, 62, 3812-3814. [CrossRef]

47. Tkachenko, S.; Datskevich, O.; Kulak, L.; Persson, C.; Engqvist, H. The effect of Al addition on the tribological behavior of Ti-Si-Zr alloys. J. Tribol. Trans. ASME 2019, 141, 041604. [CrossRef]

48. Wang, C.C.; Zhang, G.Q.; Li, Z.P.; Zeng, X.Q.; Xu, Y.; Zhao, S.C.; Hu, H.X.; Zhang, Y.D.; Ren, T.H. Tribological behavior of Ti-6Al-4V against cortical bone in different biolubricants. J. Mech. Behav. Biomed. 2019, 90, 460-471. [CrossRef]

49. Li, Y.G.; Wei, Y.H.; Hou, L.F.; Guo, C.L.; Han, P.J. Wear and electrochemical behaviour of Mg-Al intermetallic coating. Surf. Eng. 2014, 30, 933-938. [CrossRef]

50. Falat, L.; Dzupon, M.; Tavodova, M.; Hnilica, R.; L'uptacikova, V.; Ciripova, L.; Homolova, V.; Durisinova, K. Microstructure and abrasive wear resistance of various alloy hardfacings for application on heavy-duty chipper tools in forestry shredding and mulching operations. Materials 2019, 12, 2212. [CrossRef]

51. Haque, M.M.; Sharif, A. Study on wear properties of aluminium-silicon piston alloy. J. Mater. Process Tech. 2001, 118, 69-73. [CrossRef]

52. Cho, S.S.; Park, S. Finite element modeling of adhesive contact using molecular potential. Tribol. Int. 2004, 37, 763-769. [CrossRef]

(C) 2019 by the authors. Licensee MDPI, Basel, Switzerland. This article is an open access article distributed under the terms and conditions of the Creative Commons Attribution (CC BY) license (http://creativecommons.org/licenses/by/4.0/). 